\title{
Structure and activity of SLAC1 channels for stomatal signaling in leaves
}

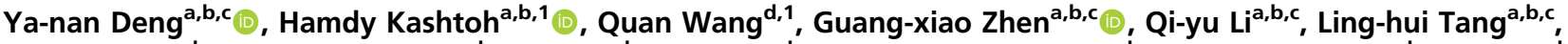

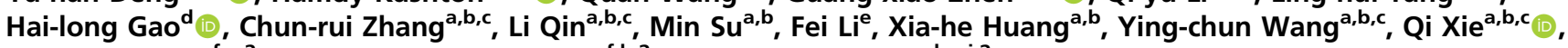 \\ Oliver B. Clarke ${ }^{f, g, 2}{ }^{-1}$, Wayne A. Hendrickson ${ }^{f, h, 2}$, and Yu-hang Chen ${ }^{a, b, c, i, 2}$
}

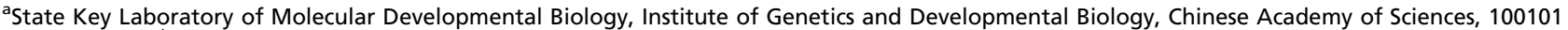
Beijing, China; 'Innovative Academy of Seed Design, Chinese Academy of Sciences, 100101 Beijing, China; 'College of Advanced Agricultural Sciences, University of Chinese Academy of Sciences, 100049 Beijing, China; ${ }^{d}$ National Laboratory of Biomacromolecules, CAS Center for Excellence in Biomacromolecules, Institute of Biophysics, Chinese Academy of Sciences, 100101 Beijing, China; ${ }^{e}$ Department of Biology, New York University, New York, NY 10003; ' Department of Physiology and Cellular Biophysics, Columbia University, New York, NY 10032; ${ }^{9}$ Department of Anesthesiology, Columbia University, New York, NY 10032; h'Department of Biochemistry and Molecular Biophysics, Columbia University, New York, NY 10032; and 'CAS Center for Excellence in Biomacromolecules, Chinese Academy of Sciences, 100101 Beijing, China
\end{abstract}

Contributed by Wayne A. Hendrickson, March 24, 2021 (sent for review July 20, 2020; reviewed by Julian I. Schroeder and Robert M. Stroud)

Stomata in leaves regulate gas exchange between the plant and its atmosphere. Various environmental stimuli elicit abscisic acid (ABA); ABA leads to phosphoactivation of slow anion channel 1 (SLAC1); SLAC1 activity reduces turgor pressure in aperture-defining guard cells; and stomatal closure ensues. We used electrophysiology for functional characterizations of Arabidopsis thaliana SLAC1 (AtSLAC1) and cryoelectron microscopy (cryo-EM) for structural analysis of Brachypodium distachyon SLAC1 (BdSLAC1), at 2.97-Å resolution. We identified 14 phosphorylation sites in AtSLAC1 and showed nearly 330-fold channel-activity enhancement with 4 to 6 of these phosphorylated. Seven SLAC1-conserved arginines are poised in BdSLAC1 for regulatory interaction with the $\mathrm{N}$-terminal extension. This BdSLAC1 structure has its pores closed, in a basal state, spring loaded by phenylalanyl residues in high-energy conformations. SLAC1 phosphorylation fine-tunes an equilibrium between basal and activated SLAC1 trimers, thereby controlling the degree of stomatal opening.

abscisic acid signaling | phosphorylation | channel activation | cryo-EM | electrophysiology

$T^{1}$ he stomatal pore, formed by a pair of specialized guard cells in plant leaves, serves as the gateway for water transpiration and atmospheric $\mathrm{CO}_{2}$ influx for photosynthesis $(1,2)$. These pores need to be tightly controlled, as inadequate $\mathrm{CO}_{2}$ intake is harmful and excessive water loss is devastating for plants (3). The guard cells respond to a wide range of environmental stimuli, including high $\mathrm{CO}_{2}$ levels, $\mathrm{O}_{3}$, low air humidity, and drought, and transduce these signals into appropriate turgor pressure changes that can adjust stomatal pore aperture $(4,5)$. It is well established that turgor pressure of guard cells is regulated by ion transport across the membrane, notably anions and potassium ions (6-8).

The phytohormone abscisic acid (ABA) plays a central role in controlling stomatal closure via activation of a complex signaling pathway that is mediated by receptors, kinase/phosphatases, and ion channels $(5,9)$. It has been known that the activation of a slow anion current in guard cells is a key event leading to stomatal closure (10-13). The corresponding gene, slow anion channel 1 (SLAC1), was discovered in genetic screens for $\mathrm{O}_{3}$ or $\mathrm{CO}_{2}$ sensitivity in Arabidopsis; when this particular channel was mutated out, the mutants showed impaired response to stimuli (14-16).

Arabidopsis thaliana SLAC1 (AtSLAC1) contains 556 amino acid (aa) residues, with a conserved transmembrane domain (TMD) between hydrophilic tails at both the $\mathrm{N}$ and $\mathrm{C}$ termini (15). Within the ABA signaling pathway, SLAC1 channel activity is controlled by a protein kinase-phosphatase pair (OST1/ABI1) (17-19). In the absence of ABA, OST1 kinase is bound and inhibited by ABI1 phosphatase (20-22). Under drought condition, ABA is accumulated into guard cells and perceived by the PYR1 receptor $(23,24)$. This resulting hormone-receptor complex then binds to phosphatase ABI1 to form a ternary hormone-receptor-phosphatase complex (25-28), which activates OST1 kinase, and leads to phosphorylation of SLAC1 $(17,18)$. Subsequently, SLAC1 releases anions $\left(\mathrm{Cl}^{-}\right.$and $\left.\mathrm{NO}_{3}{ }^{-}\right)$from guard cells, leading to membrane depolarization. Depolarization in turn activates potassium channels to release $\mathrm{K}^{+}$via $\mathrm{K}^{+}$channels and drives water out of the cell, decreasing guard cell turgor to close down the stomatal pore (8).

It has been shown that SLAC1 activation is due to phosphorylation at its $\mathrm{N}$ terminus $(17,18,29-31)$. The phosphorylation of S120 by OST1 is essential but not sufficient for SLAC1 activation monitored in Xenopus oocytes (18). S59 has been shown to be the target for both OST1 and other kinases $(29,31)$. In another phosphoproteomic analysis, using the fragment SLAC1 ${ }^{1-186}$ as substrate, S86 and S113 were also shown to be phosphorylated by OST1 (32). OST1 also phosphorylates SLAC1 at the C terminus (17), with T513 identified as a potential phosphorylation site for SLAC1 activation (31). Although multiple phosphorylation sites have been proposed, the underlying mechanism for SLAC1 activation remains elusive.

\section{Significance}

Stomatal openings in plant leaves admit $\mathrm{CO}_{2}$ and release water vapor between plant and atmosphere. Environmental stimuli such as darkness or dryness lead to closure, whereas light or low $\mathrm{CO}_{2}$ stimulate opening. Each stoma is defined by a pair of guard cells, and its pore aperture is controlled by ion channels that regulate the turgor pressure in these cells. SLAC1 anion channels are of central importance here, and environmental factors affect channel activity by controlling SLAC1 phosphorylation. Our studies define multiple sites of regulatory phosphorylation in the context of an atomic structure of the SLAC1 channel, providing a mechanistic understanding of the finetuning of channel activity and thereby of stomatal apertures in response to the environment.

Author contributions: Y.-h.C. designed research; Y.-n.D., Q.W., G.-x.Z., Q.-y.L., L.-h.T., H.-I.G., C.-r.Z., L.Q., M.S., X.-h.H., O.B.C., and Y.-h.C. performed research; Y.-n.D., H.K., F.L., Y.-c.W., Q.X., O.B.C., W.A.H., and Y.-h.C. analyzed data; and H.K., W.A.H., and Y.-h.C. wrote the paper.

Reviewers: J.I.S., University of California San Diego; and R.M.S., University of California, San Francisco.

The authors declare no competing interest.

Published under the PNAS license.

${ }^{1}$ H.K. and Q.W. contributed equally to this work.

${ }^{2}$ To whom correspondence may be addressed. Email: oc2188@cumc.columbia.edu,wah2@ cumc.columbia.edu, or yuhang.chen@genetics.ac.cn.

This article contains supporting information online at https://www.pnas.org/lookup/suppl/ doi:10.1073/pnas.2015151118/-/DCSupplemental.

Published April 29, 2021. 
Our previous study on a SLAC1 bacterial homolog from Haemophilus influenzae (HiTehA) revealed a superfamily of trimeric anion channels (33) and found each protomer gated by a highly conserved phenylalanine. We observed an unusual high-energy rotameric conformation for this gating phenylalanine (F262 in $H i$ TehA), and further mutational study on the corresponding site in AtSLAC1 (F450) suggested a unique mechanism for channel activation (33). Nevertheless, since $H i$ TehA shares a mere $19 \%$ in sequence identity with $A t$ SLAC1 and lacks the substantial $\mathrm{N}$ and $\mathrm{C}$ termini that are essential for channel activation, questions remain about SLAC1 structure and its regulation by kinase phosphorylation (7).

Here we describe the cryoelectron microscopy (cryo-EM) structure of SLAC1 from Brachypodium distachyon (BdSLAC1) at 2.97- $\AA$ resolution, and we further characterize the channel activity on Arabidopsis SLAC1. BdSLAC1 shares 63\% overall sequence identity with $A t$ SLAC1, with $73 \%$ for the pore-forming TMD and $48 \%$ for the regulatory $\mathrm{N}$ and $\mathrm{C}$ termini. As for the bacterial homolog, each $B d$ SLAC1 TMD comprises five tandemly repeated helical hairpins that surround a transmembrane pore in each protomer of the trimeric assembly. The SLAC1 pores are electropositive, consistent with its function as an anion channel. The regulatory $\mathrm{N}$-terminal domain (190 aa) and C-terminal domain (65 aa) are flexibly associated and not discernible in the structure.

We employed a SLAC1::OST1 fusion design for examining SLAC1 phosphorylation, whereby we systematically characterized critical sites responsible for channel activation. Inspired by the structure, we also conducted mutagenesis on the highly conserved phenylalanine residues related to channel gating. Altogether, our structural and functional analyses provide insights into SLAC1 gating and activity modulation. These findings allow us to propose a mechanism for finely tuned SLAC1 control of the stomatal pore in response to environmental stimuli.

\section{Results}

Biochemical Production and Structure Determination of Plant SLAC1. Fifteen plant SLAC1s were selected for gene synthesis and cloned into a modified $\mathrm{pREP} 1$ vector with capture tags for expression in the fission yeast Schizosaccharomyces pombe (34). Among those 15 SLAC1s, BdSLAC1 had the best expression and purification yield; however, it is not stable in most detergents, such as the commonly used dodecylmaltoside (DDM). Improved stability and gel-filtration profiles were achieved by using a styrene-maleic acid (SMA) polymer to extract the SLAC1 protein into SMA lipid particles (SMALPs) by using a detergent-free procedure for membrane protein solubilization (35) (SI Appendix, Fig. S1A). When solubilized in $2 \%$ SMA polymer, $B d$ SLAC1 was a suitable candidate for structural and functional characterization. No additional SMA polymer or detergents were needed to maintain the solubilized proteins in solution. We determined the structure of BdSLAC1 by cryo-EM at 2.97- $\AA$ resolution (Fig. $1 A$ and SI Appendix, Fig. S1 $B-H$ and Table S1).

The cryo-EM map for the BdSLAC1 TMD is of high quality (Fig. $2 A$ ), allowing us to build an accurate model containing residues 191 to 385 and 395 to 512 . Owing to flexibility, neither $\mathrm{N}$ - nor $\mathrm{C}$-terminal segments are resolved in the current structure. Intriguingly, perhaps as a benefit of using SMA rather than detergent for membrane extraction (36), we found densities for two lipids per protomer. Both are positioned as from the outer leaflet. Three single-chain molecules (perhaps sphingolipids from yeast) are bundled at the threefold axis of the trimer, and what seems to be a typical diglyceride binds at peripheral sites between protomers (Fig. $2 A$ and $B$ ).

Structure of Plant SLAC1. The BdSLAC1 is a symmetric trimer, and subunits are tightly associated, burying a total surface area of $\sim 6,600$ $\AA^{2}$ within interfaces. Remarkably, BdSLAC1 has positive electrostatic potential surface on both the extracellular and cytoplasmic sides (Fig. $1 B$ ), whereas bacterial HiTehA has positive charges only on the cytoplasmic surface. Each SLAC1 protomer has $10 \mathrm{TM}$ helices, adopting similar topology as HiTehA (33). They are arranged as five pairs of helical hairpins that are assembled into a unique protein fold with quasi-fivefold symmetry. $\mathrm{TM}_{\text {odd }}$ helices create a channel pore across membrane, which is blocked by a highly conserved phenylalanine residue (F460 in BdSLAC1). $\mathrm{TM}_{\text {even }}$ helices are longer and straighter, but more inclined, surrounding the inner pore and forming an outer layer (Fig. 1C). Short juxtamembrane amphipathic helices, $\mathrm{H}_{0,1}$ before $\mathrm{TM}_{1}$ and $\mathrm{H}_{2,3}$ between $\mathrm{TM}_{2}$ and $\mathrm{TM}_{3}$, are located near the $\mathrm{N}$ terminus on the cytoplasmic side.

Similar to TRIC (34), another trimeric channel, SLAC1 also has three independent pores, one through each protomer. Interestingly, highly conserved Pro or Gly residues are located in the middle of the pore-forming helices $\left(\mathrm{Gly}\right.$ in $\mathrm{TM}_{1} / \mathrm{TM}_{5}$, Pro in $\mathrm{TM}_{3} /$ $\mathrm{TM}_{7} / \mathrm{TM}_{9}$ ), which generate kinks and contribute to formation of a relatively uniform pore across membrane (Fig. $2 \mathrm{C}$ ). The pore has a diameter of $\sim 6 \AA$, except for blockage by the phenyl ring of highly conserved F460 near the middle of the membrane. Highly conserved positive charged residues, including Arg or Lys, are found near the channel pore at both cytoplasmic and extracellular sides, rendering the pore surface electropositive, consistent with SLAC1 function as an anion-conducting channel.

Despite limited sequence similarity, plant SLAC1 shares similar overall topology and trimeric assembly architecture with bacterial HiTehA (33). Nevertheless, there are distinctive differences between plant SLAC1 and bacterial TehA. When BdSLAC1 is compared to $H i$ TehA, the rmsd is $1.90 \AA$ for 269 superimposed $\mathrm{C}_{\alpha}$ of the protomer, and is $2.25 \AA$ for 793 superimposed $C_{\alpha}$ of the trimer. The most divergent regions are located near loops connecting the TM helices. A noticeable distinction is near the middle of $\mathrm{TM}_{5}$, where $H i \mathrm{TehA}$ has a $\pi$-helical turn caused by the insertion of A146 beside the P145-mediated kink (SI Appendix, Fig. S2). Another difference is at the middle of $\mathrm{TM}_{9}$ where a prolinemediated kink (P461 in BdSLAC1) replaces a water-mediated kink at a glycine residue (G263 in $H i$ TehA), but nevertheless maintains a similar conformation. Additionally, a short juxtamembrane helix $\left(\mathrm{H}_{0,1}\right)$ connects the flexible regulatory $\mathrm{N}$-terminal domain to $\mathrm{TM}_{1}$ in SLAC1 (packing against $\mathrm{H}_{2,3}$ ).

A TehA-based homology model inspired our previous SLAC1 mutagenesis for functional analysis on gating and selectivity; however, inadequate similarity between TehA and SLAC1/SLAHs also caused errors in this and other homology modeling. The alanine insertion in $\mathrm{TM}_{5}$ of TehA led to misplaced predictions of pore-lining residues in that helix $(33,37)$, and a two-residue misalignment in $\mathrm{TM}_{7}$ led to the prediction of a tryptophan residue (W404 in AtSLAC1, and W356 in AtSLAH2) as pore lining (37), whereas actually it is located opposite to the pore in BdSLAC1. Obviously having an actual SLAC1 structure provides a much improved basis for studying plant SLAC1 or SLAH channels.

SLAC1 Activation by 0ST1 Kinase. There were puzzles remaining for SLAC1 activation via OST1 phosphorylation. Coexpression of Arabidopsis SLAC1 with OST1 in Xenopus oocytes only produced weak currents, whereas their coexpression with split-YFP association (SLAC1::YFP ${ }^{\mathrm{C}}$ and OST1::YFP ${ }^{\mathrm{N}}$ ) resulted in moderate stimulation $(17,18,33)$. The poor activation of coexpression of SLAC1 and OST1 individually may be due to the lack of sufficient interaction between channel and kinase, while enhanced currents were achieved with the physical linkage from split-YFP constructs. These difficulties complicated the identification residues responsible for activating phosphorylation. To reconstitute and optimize channel activation via OST1 phosphorylation, we created a construct of SLAC1 fused directly with OST1, (SLAC1::OST1, named SOF) and performed electrophysiological measurement in oocytes after cRNA injections (Fig. $3 A$ ). We observed remarkably enhanced macroscopic current from the SLAC1::OST1 construct, 

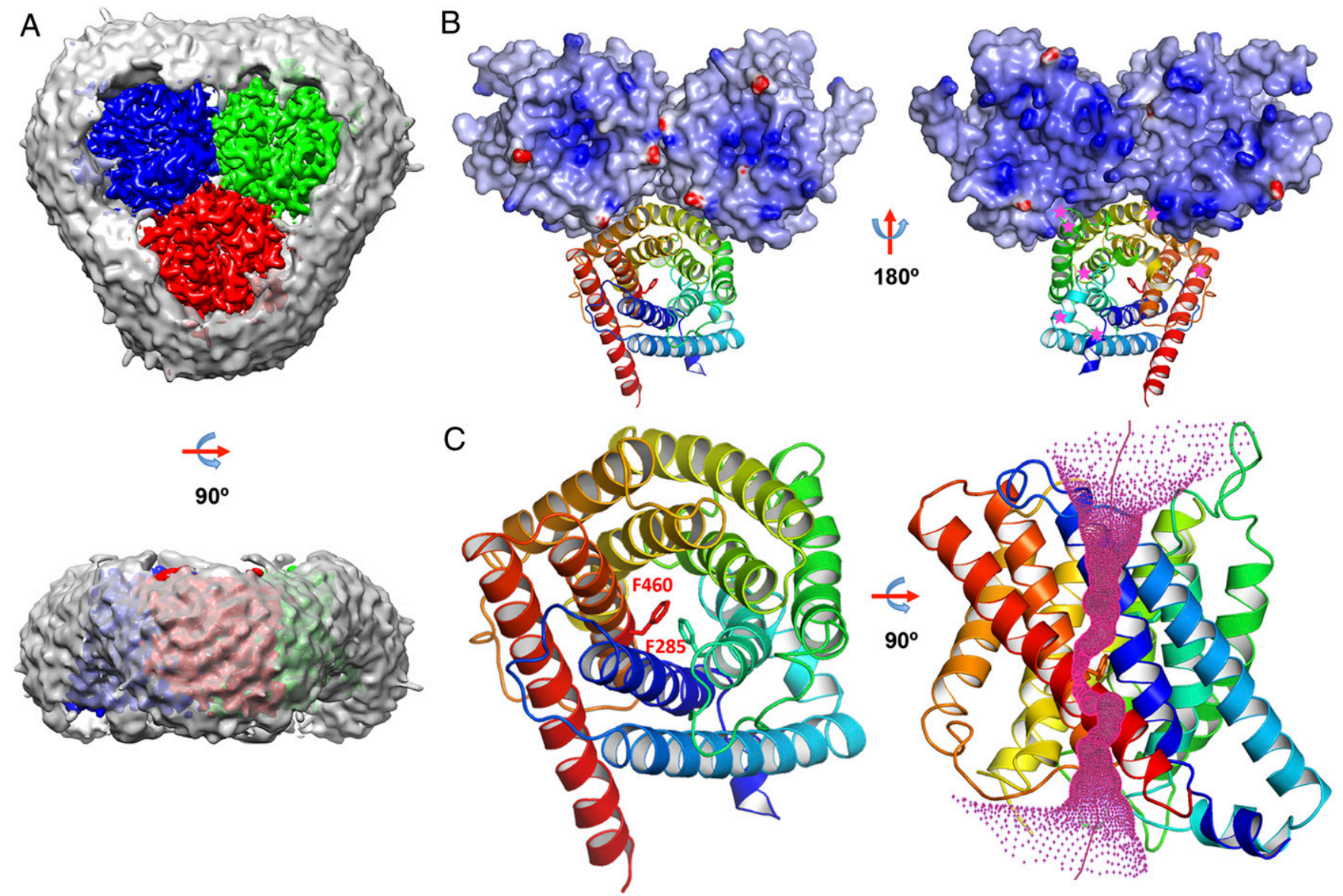

Fig. 1. Cryo-EM structure of BdSLAC1. (A) BdSLAC1 trimer embedded in SMALP, top view (Above) and side view (Below). The protomers are colored in blue, green, and red. The transparent surface indicates the boundary of the SMALP nanoparticle. (B) Electrostatic potential at the extracellular (Left) and intracellular (Right) surfaces. Electronegative and electropositive potential are colored in degrees of red and blue saturation, respectively. One protomer is drawn as a ribbon diagram and colored spectrally from blue at its $\mathrm{N}$ terminus to red at its $\mathrm{C}$ terminus. Stars indicate the position of highly conserved arginine residues (R196, R265, R272, R331, R332, R385, and R442). (C) Ribbon diagram of a BdSLAC1 protomer is colored spectrally as in B and viewed from outside (Left) and within (Right) the membrane. The side chains of F460 and F285 are shown as stick bonds within the channel pore.

much greater than in the split-YFP assay (18). To validate SLAC1 activation by OST1 phosphorylation, we disrupted the OST1 kinase activity by introducing a function impaired D160A mutation (38), and observed no apparent current for the SLAC1::OST1(D160A) construct. We further confirmed ABI1 inhibition to OST1 activity via coexpression of SLAC1::OST1 with ABI1 (SI Appendix, Fig. S3).

As our previous work suggested that conserved F450 plays an important role in channel gating, we then analyzed channel conductance properties for SLAC1 variants (wild type [WT], F450L, and F450A), in the presence and absence of OST1 and in the SLAC1::OST1 fusion construct. Compared to the coexpression of SLAC1/OST1 separately, the exceptionally large currents in SLAC1::OST1 construct suggests highly efficient channel activation. Removal of the phenyl ring in F450A SLAC1 led to increased currents both in absence and presence of OST1; however, the F450A SLAC1::OST1 current is much reduced from that with wildtype SLAC1::OST1 (Fig. $3 B$ and $C$ ). Consistent with our previous study, the F450L mutant shows negligible currents in all three cases (Fig. 3D). Puzzles from these findings are examined further below, but it seems clear that conserved gating phenylalanine F450 is crucial for SLAC1 activation.

Characterization of Phosphorylation Sites. We generated the same SLAC1::OST1 construct for expression in S. pombe. We expressed, purified, and performed mass spectrometry analyses on the fusion protein (SI Appendix, Fig. S4A). Unexpectedly, we found 14 sites of phosphorylation, including 13 in the N-terminal domain and 1 in the C-terminal segment (Fig. $4 A$ and SI Appendix, Fig. S4 $B-F$ and Table S2). Two of these sites (S59 and S120) have been characterized previously $(18,29-32)$, whereas the other 12 sites are newly identified in the current study. Whether and how these potential sites are involved in SLAC1 activation becomes an intriguing question.

To understand the role of these sites, we generated single mutants by substituting the identified candidates, serine or threonine to alanine in the SLAC1::OST1 construct, and performed conductance measurement in oocytes. We observed notable current reduction for four sites (S59, S86, S113, and S120), suggesting critical roles for these in channel activation (Fig. $4 B$ and SI Appendix, Fig. S5A). Currents were further reduced in pairwise combinations, and more still in the quadruple substitution. Moreover, the addition of T114A or/and S116A at patch 1 (S113, T114, S116, and S120) virtually abolished currents, and the patch1 quadruple mutation itself also nearly eliminated current. By contrast, the quintuple mutation at patch 2 (S134, T137, T142, S146, and S152) only slightly reduced the current (Fig. 4C). We conclude from these observations that six key sites, including S59, S86, S113, T114, S116, and S120, play important roles for SLAC1 activation by OST1 phosphorylation.

To confirm the above observation, we further designed a SLAC1::OST1 mutant construct (named SLAC1::OST1-13A or SOF-13A), which replaced all 13 phosphorylation sites in the 

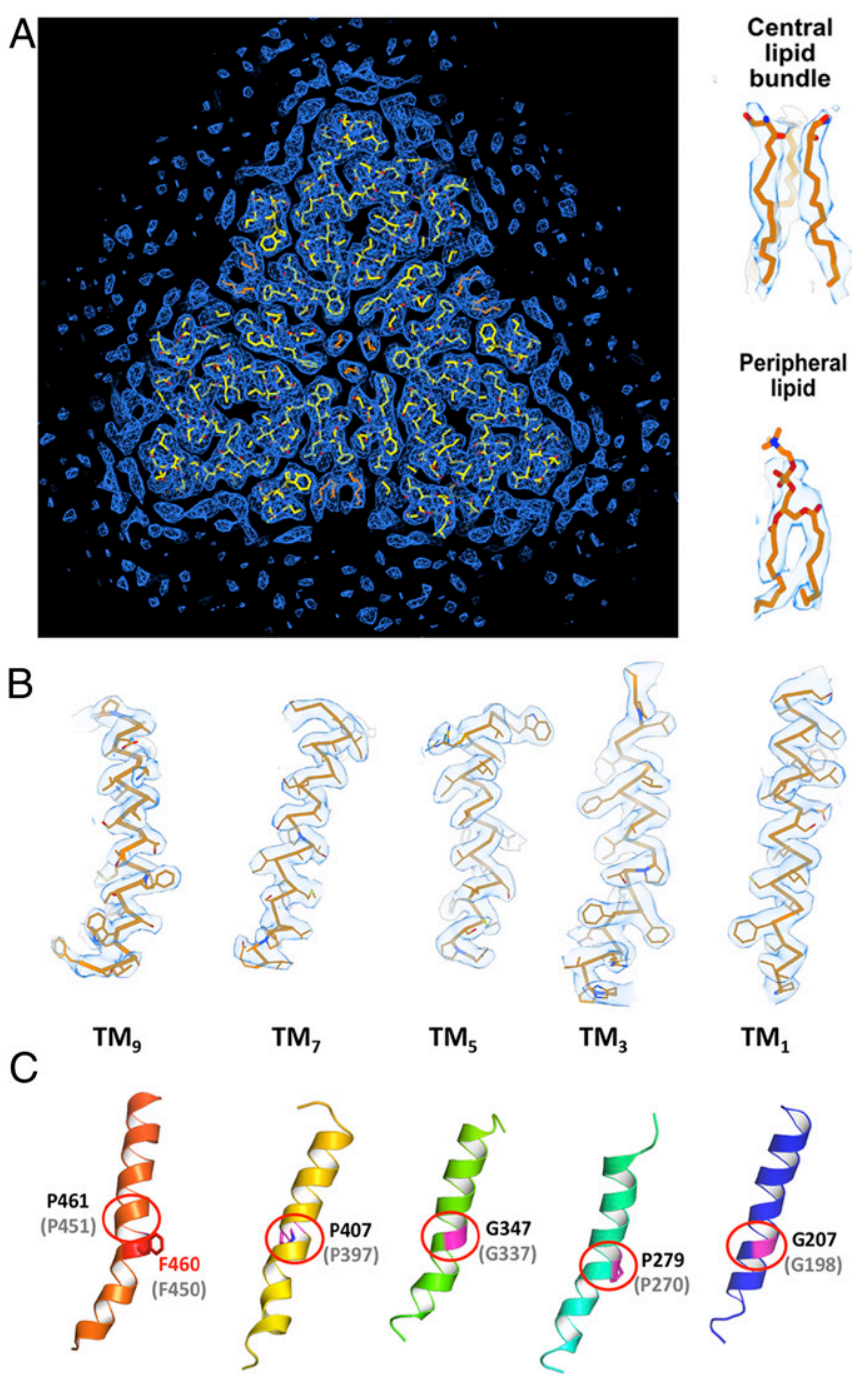

$\mathrm{TM}_{5}$ $\mathrm{TM}_{3}$
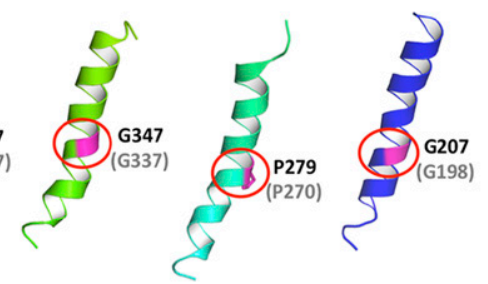

Fig. 2. The cryo-EM map for BdSLAC1. (A) Density distribution from $B d S L A C 1$ cryo-EM at $2.97 \AA$ and superimposed model (protein, yellow; lipids, orange). The central lipid bundle and the peripheral lipid are shown. $(B)$ Representative cryo-EM density map for the $\mathrm{TM}_{\text {odd }}$ segments. The helices are drawn from the unrolled pore, as oriented in Fig. 1C. The maps are contoured at a level of 0.67 , and density is shown within a $2.5-\AA$ zone around the atoms belonging to each segment, using the "sop zone" command in UCSF Chimera. (C) Kinks in the pore-forming $\mathrm{TM}_{\text {odd }}$ helices, orientated as in B. The kink-mediated Pro or Gly residues (G207 in TM1, P279 in TM3, G347 in TM5, P407 in TM7, and P461 in TM9) are shown and colored magenta.

N-terminal domain to alanine residues. The SOF-13A shows little current activity; however, substantial current was restored upon removal of the phenyl ring of F450 in SOF-13A_F450A, compared to the SLAC1 F450A mutant. This demonstrates that the lack of current in SOF-13A is due to loss of phosphorylation, rather than failure of surface expression in oocytes (SI Appendix, Fig. S5B). This observation also suggests that the reduced currents of other such mutants (singly and in combination) most likely reflect impaired channel activity due to the reduced phosphorylation. Next, we examined functional significance of the six key residues by restoring them (singly or in combination) back into the SOF-13A construct (Fig. 4D). Among these, only A113S shows large current, which is enhanced appreciably for the A86S/A113S double restoration. SLAC1 activity was completely restored when four to six key phosphorylation sites were restored back into SOF-13A (Fig. $4 D$ ). These results confirm the critical roles for these six key sites in SLAC1 activation, and combined phosphorylation of multiple sites potentiates SLAC1 to full activity.

Additionally, we created phosphomimetic mutants for the six key sites by substitution to aspartate residues. Single phosphomimetics did not produce noticeable currents, whereas combinations of two to six sites gradually stimulated channel activities to a substantial level (Fig. $4 E$ and SI Appendix, Fig. S5 $C$ and D). Overall, these results suggest synergetic phosphorylation of multiple sites can provide a fine-tuning mechanism for modulating various SLAC1 activities.

Some puzzles remain regarding C-terminal phosphorylation sites. Although S543 was phosphorylated in the mass spectrometry analyses, we observed no current reduction upon substitution to alanine in the SLAC1::OST1 construct, and found no stimulation for S543D SLAC1. On the other hand, another study showed that phosphomimetic mutant T513D stimulated currents (31), even though we detected no phosphorylation in our experiment. We reproduce the finding on $\mathrm{T} 513 \mathrm{D}$ and also fail to observe phosphorylation on T111, a previously unreported site. T111A substitution showed no current reduction in SLAC1::OST1, but the phosphomimetic mutant T111D did stimulate currents in SLAC1 (SI Appendix, Fig. S5 $E$ and $F$ ). These observations indicate that both T111 and T513 may play important roles in channel activation; yet further investigation is needed.

SLAC1 Pore and Gating Mechanism. Little is known about how SLAC1 phosphorylation is coupled to channel gating, but new clues come from structural features of pore-lining residues in our current structure. The SLAC1 pore is lined with highly conserved and generally hydrophobic residues (Fig. $5 A$ and $B$ ). The SLAC superfamily shares an extremely conserved gating phenylalanine (F450 in AtSLAC1, F460 in BdSLAC1, and F262 in HiTehA). In $H i$ TehA, the phenyl ring (F262) is restricted in a high-energy conformation $\left(-160^{\circ} /-4^{\circ}\right.$ for $\left.\chi 1 / \chi 2\right)$, by contacts with neighboring L18 $\left(\mathrm{TM}_{1}\right)$ and V210 $\left(\mathrm{TM}_{7}\right)(33)$. The corresponding residues in BdSLAC1 are L206 and A409, respectively; and both are conserved in plant SLAC1s. The replacement of Val by Ala would partially release the constraint on the gating phenylalanine. In $B d$ SLAC1, the corresponding gating phenylalanine F460 also adopts a strained conformation $\left(159^{\circ} / 47^{\circ}\right.$ for $\left.\chi 1 / \chi 2\right)$. We found that another pore-lining phenylalanine (F285 in BdSLAC1, TM $\left.{ }_{3}\right)$ lays in a close van der Waals contact with the gating phenylalanine F460, having a $\chi 1 / \chi 2$ angle of $-94^{\circ} /-31^{\circ}$. Both phenylalanine residues are highly conserved in plant SLAC1s (F267 and F450 in AtSLAC1). Different from bacterial HiTehA, these dual phenyl rings interact and render both in energy unfavorable conformations, suggesting a critical role in channel gating (Fig. 5C). In addition, the kink at the middle of $\mathrm{TM}_{9}$ in $H i \mathrm{TehA}$ (mediated by G263 and a water molecule) is replaced by a proline-mediated kink in SLAC1 (P451 in AtSLAC1 and P461 in BdSLAC1).

Taken together, distinguishing structural characteristics of the $B d$ SLAC1 pore indicate that the mechanism for channel activation in plant SLAC1 is distinctive from that in bacterial homologs. The conformation of proline-kinked $\mathrm{TM}_{9}$ in SLAC1 is similar to that with the water-mediated kink in TehA (SI Appendix, Fig. $\mathrm{S} 6 A$ ), but the distinctions are such that they impact pore geometry and likely affect conductance. In examining the gating mechanism in Arabidopsis SLAC1, we first substituted P451 with glycine (as in bacterial TehA) and also alanine for testing in SLAC1::OST. These mutations reduced channel currents markedly, respectively, to $36 \%$ and $24 \%$ of wild type (SI Appendix, Fig. S6 B and $C$ ), likely due to impaired channel activities or possibly from altered trafficking to the plasma membrane.

We also mutated the pore phenylalanine residues, F276 and F450, to alanine in both SLAC1 and SLAC1::OST, and measured resulting conductance in oocytes (SI Appendix, Fig. S7). Compared to the small but significant current in the wild type, 

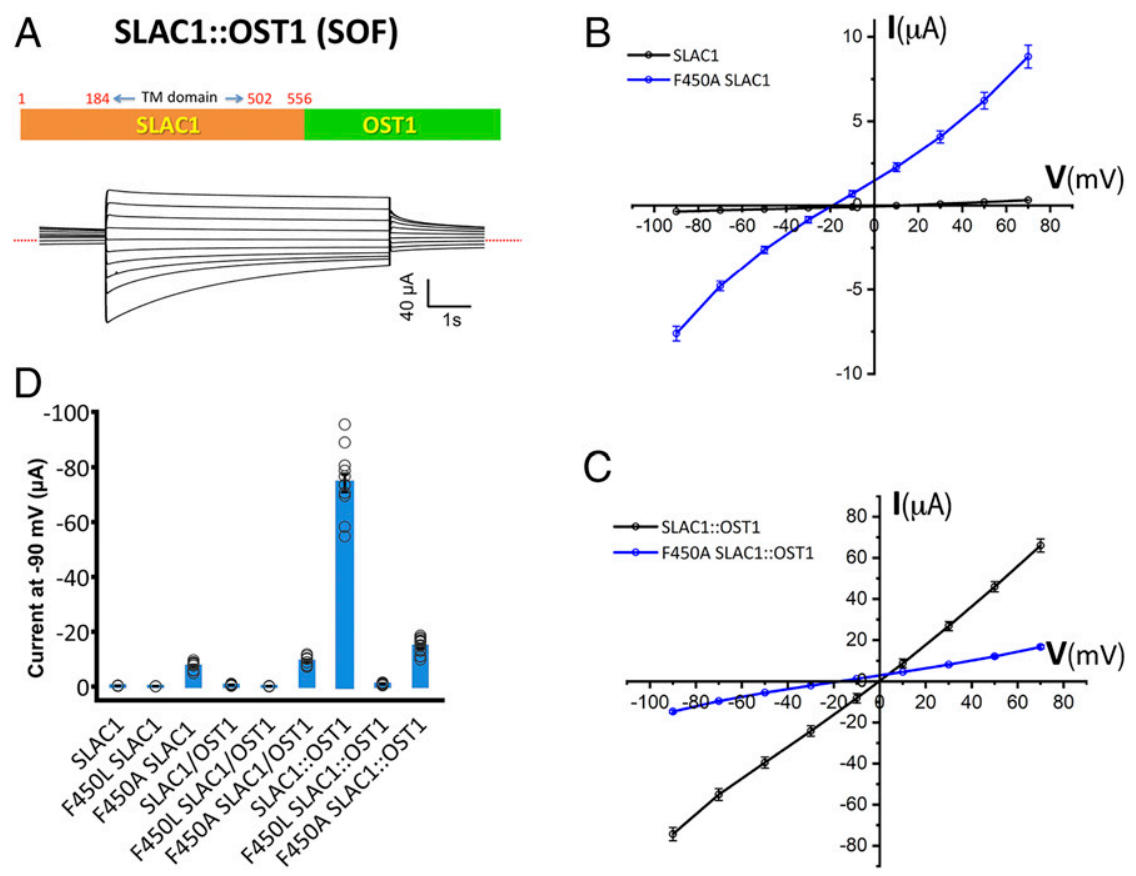

Fig. 3. Electrophysiological characterization of $A t S L A C 1:: O S T 1 .(A, A b o v e)$ Construct design for SLAC1::OST1 (SOF); AtSLAC1 is fused directly with AtOST1. (Below) Typical Xenopus oocyte microelectrode voltage-clamp current traces ( $-110 \mathrm{mV}$ to $70 \mathrm{mV}$ in $20 \mathrm{mV}$ steps). (B and C) Current-voltage (I-V) relations for Arabidopsis SLAC1 wild type, F450A mutant. Note different $y$ axis scales. (D) Effects of gating residue mutations. Mean chloride currents, measured at - 90 mV, are shown comparing WT AtSLAC1 and its variants (F450A and F450L), alone, coexpressed with OST1, and SLAC1::OST1.

the F276A mutant showed enhanced leaky conductance with altered properties in the absence of OST1, suggesting a role for F276 in channel gating (Fig. 5D). The roles of F276 and F450 are complicated, however. The F276A, F450A, and F276A/F450A mutants all increased currents relative to wild type when in unphosphorylated SLAC1, by $154 \%, 2100 \%$, and $668 \%$, respectively, whereas they decreased currents to $62 \%, 21 \%$, and $58 \%$ of wild type, respectively, when in the SLAC1::OSTactivated channel (Fig. 5E).

There is no simple explanation for the results from porephenylalanine mutations; effects of the phenyl ring deletions are opposite in unphosphorylated and phosphorylated channels. The pore as restructured after activation must differ substantially, and at present indecipherably, from the $B d \mathrm{SLAC} 1$ basal-state pore. It makes sense from the basal-state structure that the current should be greatly enhanced when the phenyl ring is removed in F450A, but the substantial reduction of that current in the F276A/F450A double mutant implies added conformational change. The relatively modest increase of current upon phosphorylation of F450A (3.3-fold) compared to the $~ 330$-fold increase for the WT channel (Fig. 3D) suggests radical change. Furthering the complications, another study has found that F450A leads to large currents in oocytes, but not in the absence of OST1 (39); this latter observation conflicts with our findings here and those reported previously (33). Activation might plausibly entail pore-opening quaternary rearrangements in the WT trimer, and the propensity for such activation might differ for different mutants. Indeed, the virtually complete loss of phosphoactivation in the F450L channel may simply mean that this mutant cannot undergo conformational activation.

\section{Discussion}

Plants must maintain a delicate balance between gas exchange necessary for photosynthesis and water conservation in the surrounding environment, which they do by regulating stomatal pores in the leaf epidermis. Environmental factors, such as elevated $\mathrm{CO}_{2}$ levels, darkness, and drought, activate SLAC1 channels to induce stomatal closure through reduction of turgor pressure in guard cells. The SLAC1 activation is controlled largely by OST1 phosphorylation (6-8), and SLAC1 activity adjusts the turgor pressure in guard cells and thereby controls stomatal apertures; however, the underlying mechanism for SLAC1 phosphoactivation is largely unknown.

Following the leads of others, we have studied SLAC1 activity by expression in Xenopus oocytes to gain insight into OST1 phosphorylations of SLAC1, recognizing that SLAC1 regulation in plant cells can differ from that obtained in oocytes. The complement of protein kinases and other relevant factors in plant guard cells likely differs from those present in oocytes, and differing SLAC1 channels may be presented differently onto oocyte plasma membranes. Nevertheless, despite the reservations, we have gained useful insights from electrophysiology in oocytes that complement our structural analysis. Here too, we face limitations. We were able to perform the functional studies with Arabadopsis SLAC1 and the structural analysis on Brachypodium SLAC1; and, whereas ideally we would have both functional and structural data from the very same SLAC1 molecules, we must rely on the high sequence similarity (63\% aa identity overall, $73 \%$ for the transmembrane domain) in drawing conclusions.

Previous studies in Xenopus oocytes showed that OST1 alone barely activates SLAC1, usually at the level significantly less than those observed by CDPKs or CIPKs. A possible explanation could be weak or transient interaction between SLAC1 and OST1, perhaps due to the lack of an unknown mediating factor in the heterologous system. Indeed, when OST1 and SLAC1 were drawn together through split YFP association (SLAC1::YFPC and OST1::YFP ${ }^{\mathrm{N}}$ ), SLAC1 activation via OST1 phosphorylation in oocytes was enhanced substantially (18). We suspected that the split YFP encounters may not have been complete, and, so we took away the problem by using the direct fusion SLAC1::OST1 to assure avidity. Thereby, OST1 phosphorylation enhanced AtSLAC1 channel activity $\sim 330$-fold (Fig. $3 D$ ). Another explanation for low SLAC1 activation by simple coexpression of SLAC1 and OST1 has recently come to light from work of Takahashi et al. (40), suggesting that MAPKKK kinase can activate OST1 by phosphorylating OST1 
A

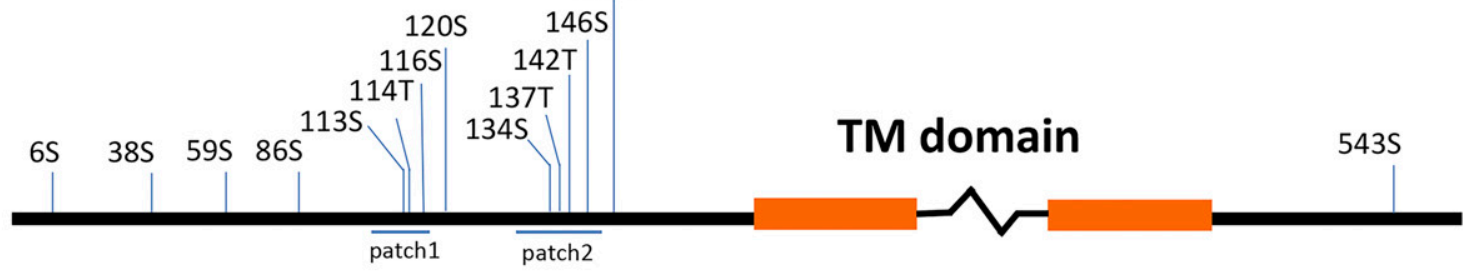

B

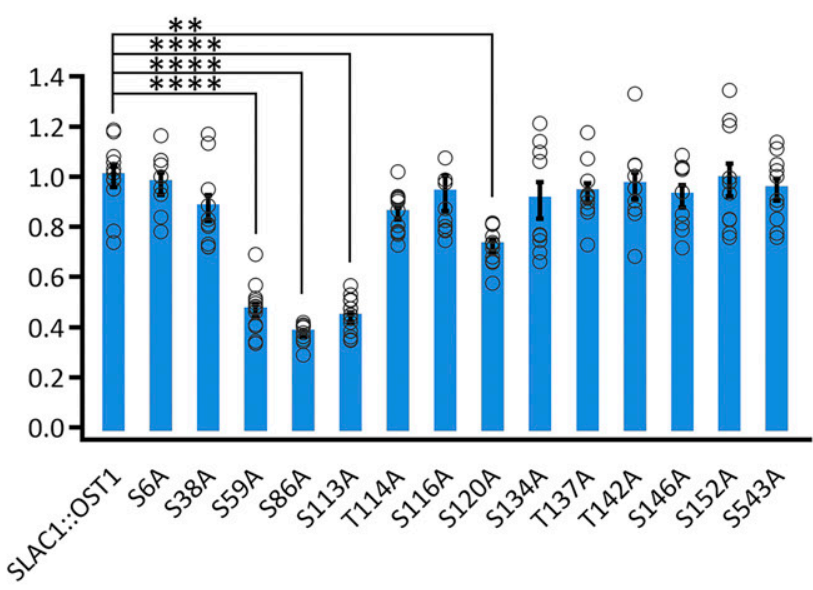

C
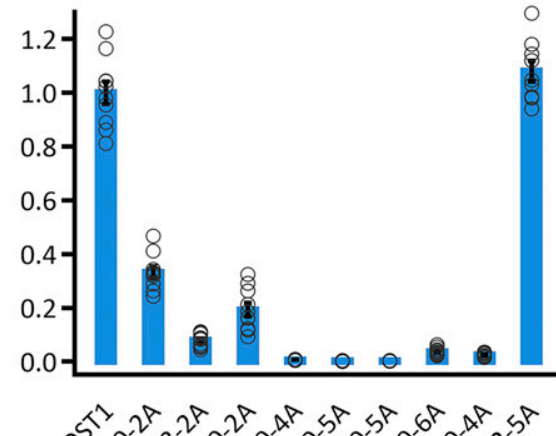

कर दो

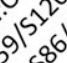

D

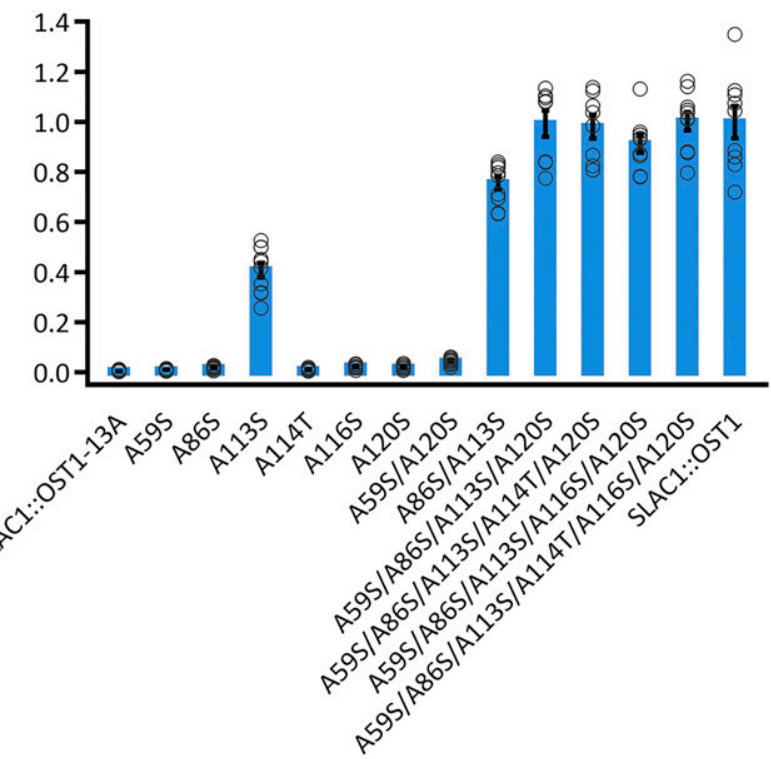

E

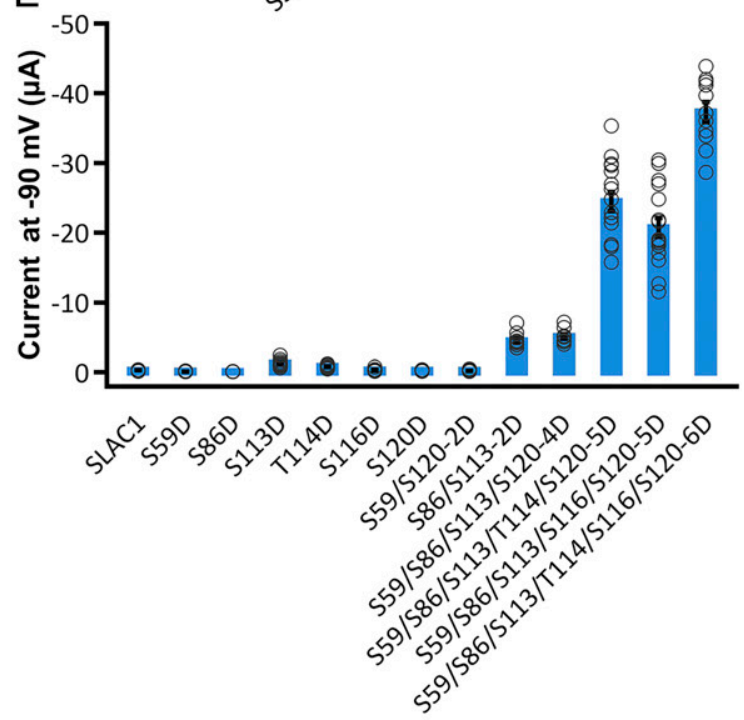

Fig. 4. Characterization of phosphorylation sites in AtSLAC1. (A) Phosphorylation sites identified in mass spectrometry by using SLAC1::OST1. All phosphorylated residues are indicated, 13 at the $\mathrm{N}$ terminus and 1 at the $\mathrm{C}$ terminus. (B) Effects of single alanine mutations in SLAC1::OST1. Mutations S59A, S86A S113A, and S120A resulted in substantially reduced currents. The asterisks indicate significance of one-way ANOVA analyses and Tukey's test: $* * P<0.01$ and $* * * * P<0.0001$. (C) Effects of mutant combinations in SLAC1::OST1. Double mutants (S59A/S120A, S86A/S113A, and S113A/S120A) and quadruple mutant (S59A/S86A/S113A/S120A) significantly reduced currents, and additions of such S/T-A mutations to that quadruple mutant essentially eliminated currents. The patch-1 quadruple mutant (S113A/T114A/S116A/S120A) almost abolished channel current, whereas the patch-2 quintuple mutant (S134A/T137A/T142A S146A/S152A) showed no effect. (D) Effects of restoring key sites (S59, S86, S113, T114, S116, and S120) back into SLAC1-13A::OST1. The SLAC1-13A::OST1 construct contains 13 alanine mutations for the identified phosphorylation N-terminal-doman residues, and serves as a "blank" control construct. (E) Electrophysiological analyses of SLAC1 phosphomimetics. Aspartate substitutions of two to six sites gradually restore channel activities to a substantial level (in the absence of OST1) (mean \pm SEM, $n>8$ ). 
A

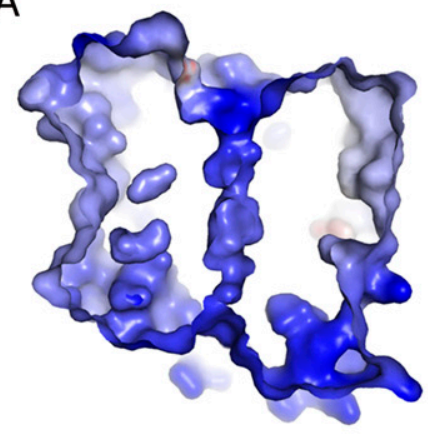

C

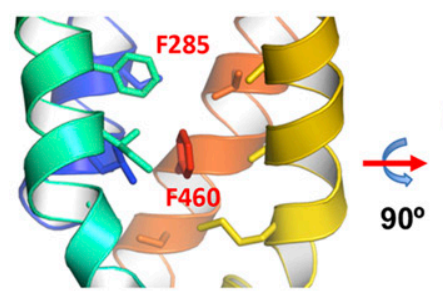

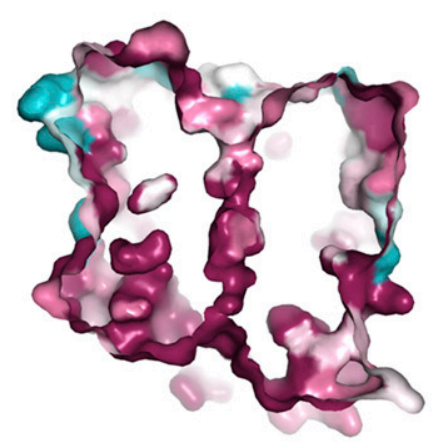

B
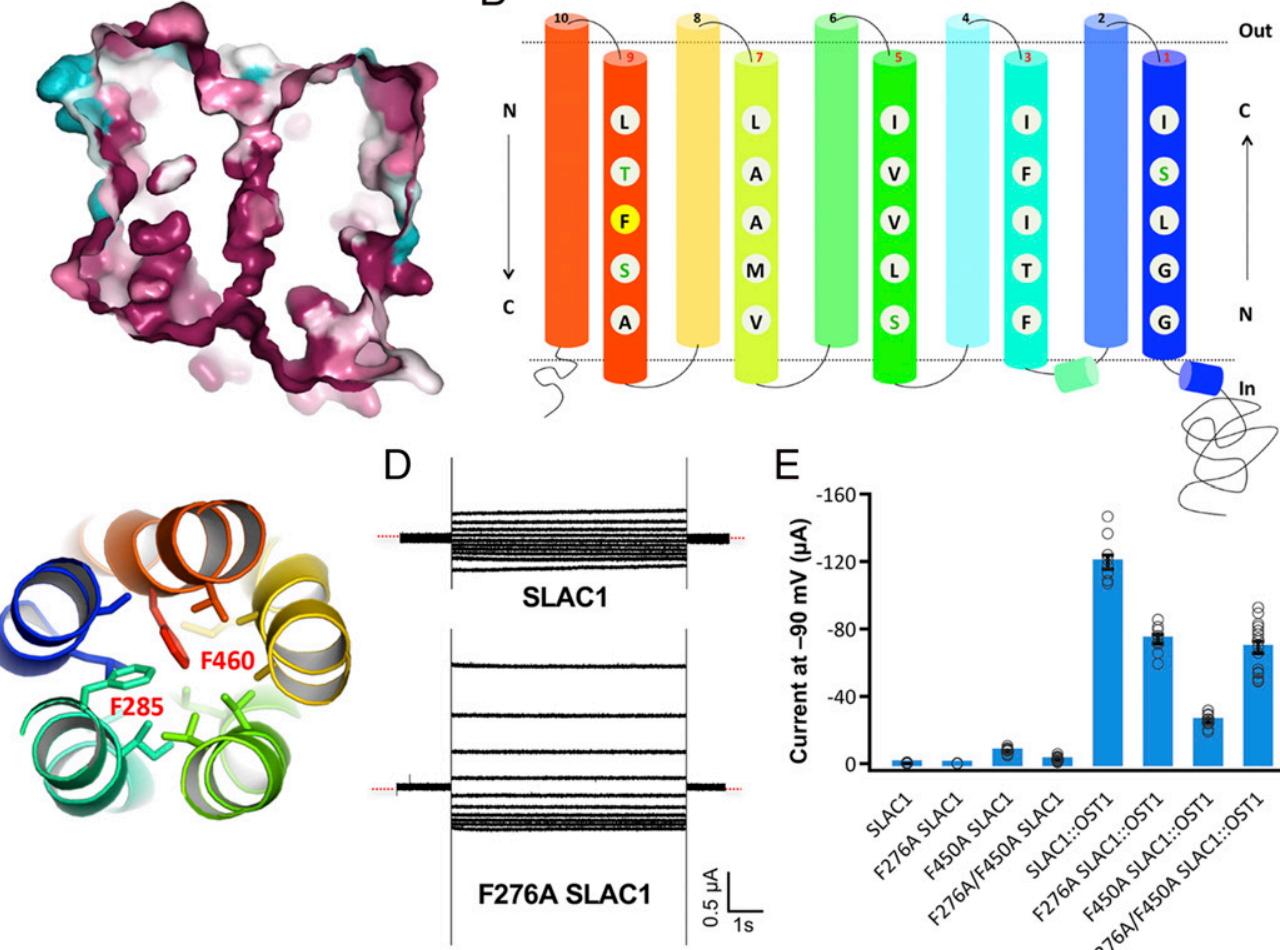

E

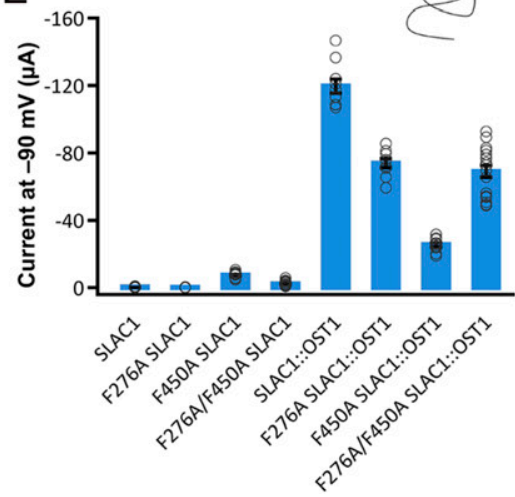

Fig. 5. The SLAC1 channel gating. (A) Cross-section through the model of BdSLAC1 F460A. The model is oriented as in Fig. 1C, with the electrostatic potential (Left)

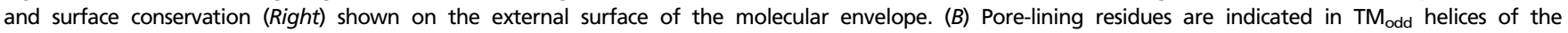
BdSLAC1 rolled-open model. (C) Molecular basis for conformational strain between the highly conserved pore lining phenyl residues F460 and F285 (respectively,

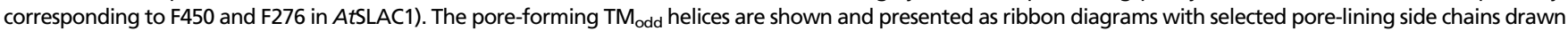
in stick representation, top view (Left) and side view (Right, with $\mathrm{TM}_{5}$ removed). The dual phenyl rings interact and render both in energetically unfavorable conformations, with $\chi 1 / \chi 2$ at $-94^{\circ} /-31^{\circ}$ (F285) and $159^{\circ} / 47^{\circ}$ (F460), suggesting critical roles in channel gating. (D) Representative current traces of SLAC1 and F276 SLAC1. (E) Mean chloride currents, measured at $-90 \mathrm{mV}$, are shown comparing WT, F276A, F450A, and F276AF450A mutant, in the absence of OST1 and in SLAC1::OST1.

S171; however, native background MAPKKK activity seems to be missing in Xenopus oocytes. Coincidentally, we found that OST1 S171 was phosphorylated in the SLAC1::OST1 fusion when expressed in S. pombe (SI Appendix, Table S2).

The present study appreciably advances understanding of the mechanism for phosphoactivation of SLAC1. Besides obtaining an atomic-level picture of $B d$ SLAC1, we identified 14 sites of phosphorylation by using an AtSLAC1::OST1 fusion construct to enhance avidity of the kinase-substrate interaction. Among them, six residues located in the flexible N-terminal domain (S59, S86, S113, T114, S116, and S120) all play key roles in activating the channel. Whether and which of these sites are phosphorylated in vivo in plants, by OST1 and other kinases, needs further investigation. It is also unclear whether any phosphorylation occurs in the SLAC1 transmembrane domain, from which we have not observed any phosphorylated peptides in the mass spectrum of the purified SLAC1::OST1 protein.

Phosphorylation is one of the most significant of posttranslational modifications, used to modulate channels and transporters in many signaling pathways and by diverse mechanisms. Some transport proteins are inactive when their cytoplasmic loops/tails block the channel pore at resting state $(41,42)$. Phosphorylation usually induces conformational changes to release inhibiting blockage in these proteins, and in turn renders them active. Phosphorylation of plant aquaporin (SoPIP2) rearranges its cytoplasmic gate-blocking loop to open the channel (41). Another example is the CFTR chloride channel, for which phosphorylation disengages its regulatory domain from an inhibitory position and leads to channel opening (42). In yet another case, phosphorylation switches the dual-affinity nitrate transporter NRT1.1 from the low-to-high-affinity mode by decoupling the dimer and increasing structure flexibility $(43,44)$. What then is the basis for SLAC1 channel activation after phosphorylation? As we have seen, efficacious SLAC1 phosphorylation sites are at AtSLAC1 positions 59, 86, and a patch of four residues among 113 to 120, each imbedded in an island of conservation; yet, despite especially strong juxta-TMD conservation (BdSLAC1 residues 154 to 188), the entire N-terminal domain (1 to 188) is invisible in the current $B d \mathrm{SLAC1}$ structure and presumed to be flexibly associated. Thus, the actual mechanism of SLAC1 phosphoregulation remains elusive.

In principle, we contemplate two alternative mechanisms: one a binding-activation model in which phosphorylated domains interact with the TMD to exert pore-opening conformational changes, and the second an inhibition-release model in which interactions that hold the channel in a closed state would be released upon phosphorylation. We initially disfavored the latter since baseline channel activity and stomatal openings are the same for $\Delta N / \Delta C$ SLAC1 as for WT SLAC1 (45), even though ABA responsiveness was abolished, and because the N-terminal domain is only flexibly associated and not TMD bound in the cryo-EM structure. Moreover, as for the binding-activation model, we could imagine phosphorylated N-terminal domain residues being attracted to the positively charged cytoplasmic TMD surface of SLAC1, which features seven arginine residues that are identical in all SLAC1identified sequences (R187, R256, R263, R321, R322, R375, and R432 in AtSLAC1) and five sites having either lysine or arginine residues at $>94 \%$ incidence $(\mathrm{K} 255, \mathrm{~K} 320, \mathrm{~K} 325, \mathrm{~K} 384$, and K440 in 
AtSLAC1). Nevertheless, consistent with an inhibition-release model, we have recently observed robust kinase-independent SLAC1 activation after alternative N-terminal truncations. It may be that actual associations of $\mathrm{N}$-terminal domain segments with the TMD were displaced due to air-water interface effects during cryoEM sample preparation. We continue to seek conditions for capturing open-pore SLAC1 structures.

Puzzles that remain for these and other future investigations include the conformational changes that SLAC1 undergoes upon phosphorylation, the mechanism by which the phosphorylated N-terminal domain of SLAC1 promotes the activated conformation, possible allosteric modulation by bicarbonate (39) and other ligands, whether calcium-dependent kinases and other kinases play complementary as well as overlapping roles, the role of lipids in channel stability and/or activity, and the structural basis for anion selectivity. Deep insight into the mechanism of SLAC1 control on stomatal pores will be valuable for designing drought-tolerant or water-efficient plant cultivation.

\section{Materials and Methods}

Protein Production and Structure Determination. A structural genomics approach, screening 15 plant species, was used to identify BdSLAC1 as being suitable for expression in S. pombe for use in structure determination. Purified BdSLAC1 was applied to gold grids, frozen in a TFS Vitrobot, and micrographs were collected in a Titan Krios equipped with a K2 detector. Images were motion corrected, particles were picked and classified, three-

1. A. M. Hetherington, F. I. Woodward, The role of stomata in sensing and driving environmental change. Nature 424, 901-908 (2003).

2. C. Sirichandra, A. Wasilewska, F. Vlad, C. Valon, J. Leung, The guard cell as a single-cell model towards understanding drought tolerance and abscisic acid action. J. Exp. Bot. 60 1439-1463 (2009).

3. J. I. Schroeder, J. M. Kwak, G. J. Allen, Guard cell abscisic acid signalling and engineering drought hardiness in plants. Nature 410, 327-330 (2001).

4. T.-H. Kim, M. Böhmer, H. Hu, N. Nishimura, J. I. Schroeder, Guard cell signal transduction network: Advances in understanding abscisic acid, $\mathrm{CO}_{2}$, and $\mathrm{Ca}^{2+}$ signaling. Annu. Rev. Plant Biol. 61, 561-591 (2010).

5. S. Munemasa et al., Mechanisms of abscisic acid-mediated control of stomatal aperture. Curr. Opin. Plant Biol. 28, 154-162 (2015).

6. H. Kollist, M. Nuhkat, M. R. G. Roelfsema, Closing gaps: Linking elements that control stomatal movement. New Phytol. 203, 44-62 (2014).

7. R. Hedrich, D. Geiger, Biology of SLAC1-type anion channels-From nutrient uptake to stomatal closure. New Phytol. 216, 46-61 (2017).

8. R. Hedrich, lon channels in plants. Physiol. Rev. 92, 1777-1811 (2012).

9. Y. Murata, I. C. Mori, S. Munemasa, Diverse stomatal signaling and the signal integration mechanism. Annu. Rev. Plant Biol. 66, 369-392 (2015).

10. J. I. Schroeder, S. Hagiwara, Cytosolic calcium regulates ion channels in the plasma membrane of Vicia faba guard cells. Nature 338, 427-430 (1989).

11. S. Pandey, W. Zhang, S. M. Assmann, Roles of ion channels and transporters in guard cell signal transduction. FEBS Lett. 581, 2325-2336 (2007).

12. M. R. G. Roelfsema, R. Hedrich, D. Geiger, Anion channels: Master switches of stress responses. Trends Plant Sci. 17, 221-229 (2012).

13. S. Saito, N. Uozumi, Guard cell membrane anion transport systems and their regulatory components: An elaborate mechanism controlling stress-induced stomatal closure. Plants 8, 9 (2019).

14. J. Negi et al., $\mathrm{CO}_{2}$ regulator SLAC1 and its homologues are essential for anion homeostasis in plant cells. Nature 452, 483-486 (2008).

15. T. Vahisalu et al., SLAC1 is required for plant guard cell S-type anion channel function in stomatal signalling. Nature 452, 487-491 (2008).

16. S. Saji et al., Disruption of a gene encoding C4-dicarboxylate transporter-like protein increases ozone sensitivity through deregulation of the stomatal response in Arabidopsis thaliana. Plant Cell Physiol. 49, 2-10 (2008).

17. S. C. Lee, W. Lan, B. B. Buchanan, S. Luan, A protein kinase-phosphatase pair interacts with an ion channel to regulate ABA signaling in plant guard cells. Proc. Natl. Acad. Sci. U.S.A. 106, 21419-21424 (2009).

18. D. Geiger et al., Activity of guard cell anion channel SLAC1 is controlled by drought-stress signaling kinase-phosphatase pair. Proc. Natl. Acad. Sci. U.S.A. 106, 21425-21430 (2009)

19. A. C. Mustilli, S. Merlot, A. Vavasseur, F. Fenzi, J. Giraudat, Arabidopsis OST1 protein kinase mediates the regulation of stomatal aperture by abscisic acid and acts upstream of reactive oxygen species production. Plant Cell 14, 3089-3099 (2002).

20. F. F. Soon et al., Molecular mimicry regulates ABA signaling by SnRK2 kinases and PP2C phosphatases. Science 335, 85-88 (2012).

21. J. Leung et al., Arabidopsis $A B A$ response gene $A B I 1$ : Features of a calcium-modulated protein phosphatase. Science 264, 1448-1452 (1994).

22. K. Meyer, M. P. Leube, E. Grill, A protein phosphatase $2 \mathrm{C}$ involved in ABA signal transduction in Arabidopsis thaliana. Science 264, 1452-1455 (1994). dimensional (3D) reconstructions were performed, and the structure was built and refined as described in SI Appendix.

Electrophysiology. Channel properties were analyzed for AtSLAC1 and selected mutant variants after injection of corresponding cRNAs into Xenopus oocytes as before (33).

Mass Spectrometry. Protein bands were excised from SDS-polyacrylamide gel electrophoresis (SDS-PAGE) gels, digested with trypsin, and peptides were analyzed by liquid chromatography-tandem mass spectrometry using Linear Trap Quadropole-Orbitrap elite mass spectrometer.

Data Availability. All study data are included in the article and/or SI Appendix. The accession number for the 3D cryo-EM density map at the Electron Microscopy Data Bank (EMDB) is EMD-31197, and the Protein Data Bank (PDB) accession code for the coordinates is 7ENO.

ACKNOWLEDGMENTS. We thank Dr. Guang-zhe Yang for suggestions on electrophysiological experiments and De-lin Li, Ling-min Yuan, and other colleagues for expression screening in the early stages of the project. We are also grateful to the staff at the Center for Biological Imaging, Institute of Biophysics, Chinese Academy of Sciences. This project was financially supported by the National Key Research and Development Program of China (Grants 2016YFA0500503 and 2020YFA0509903), the Strategic Priority Research Program of the Chinese Academy of Sciences (Precision Seed Design and Breeding, Grant XDA24020305), and the National Natural Science Foundation of China (Grants 31470728, 31322005, and 31872721) to Y.-h.C., by NIH Grant R35GM134920-01 and NSF Grant MCB-1934628 to F.L., and by NIH Grants R01GM107462 and P41GM116799 to W.A.H.

23. Y. Ma et al., Regulators of PP2C phosphatase activity function as abscisic acid sensors Science 324, 1064-1068 (2009).

24. S.-Y. Park et al., Abscisic acid inhibits type $2 \mathrm{C}$ protein phosphatases via the PYR/PYL family of START proteins. Science 324, 1068-1071 (2009).

25. K. Melcher et al., A gate-latch-lock mechanism for hormone signalling by abscisic acid receptors. Nature 462, 602-608 (2009).

26. K. Miyazono et al., Structural basis of abscisic acid signalling. Nature 462, 609-614 (2009).

27. H. Fujii et al., In vitro reconstitution of an abscisic acid signalling pathway. Nature 462 660-664 (2009)

28. N. Nishimura et al., PYR/PYL/RCAR family members are major in-vivo ABI1 protein phosphatase 2C-interacting proteins in Arabidopsis. Plant J. 61, 290-299 (2010)

29. B. Brandt et al., Reconstitution of abscisic acid activation of SLAC1 anion channel by CPK6 and OST1 kinases and branched ABI1 PP2C phosphatase action. Proc. Natl. Acad. Sci. U.S.A. 109, 10593-10598 (2012)

30. B. Brandt et al., Calcium specificity signaling mechanisms in abscisic acid signal transduction in Arabidopsis guard cells. eLife 4, 1-25 (2015)

31. T. Maierhofer et al., Site- and kinase-specific phosphorylation-mediated activation of SLAC1, a guard cell anion channel stimulated by abscisic acid. Sci. Signal. 7, ra86 (2014).

32. T. Vahisalu et al., Ozone-triggered rapid stomatal response involves the production of reactive oxygen species, and is controlled by SLAC1 and OST1. Plant J. 62, 442-453 (2010).

33. Y.-H. Chen et al., Homologue structure of the SLAC1 anion channel for closing stomata in leaves. Nature 467, 1074-1080 (2010)

34. X.-H. Wang et al., Structural basis for activity of TRIC counter-ion channels in calcium release. Proc. Natl. Acad. Sci. U.S.A. 116, 4238-4243 (2019).

35. S. C. Lee et al., A method for detergent-free isolation of membrane proteins in their local lipid environment. Nat. Protoc. 11, 1149-1162 (2016).

36. W. Qiu et al., Structure and activity of lipid bilayer within a membrane-protein transporter. Proc. Natl. Acad. Sci. U.S.A. 115, 12985-12990 (2018).

37. T. Maierhofer et al., A single-pore residue renders the Arabidopsis root anion channe SLAH2 highly nitrate selective. Plant Cell 26, 2554-2567 (2014).

38. C. Yunta, M. Martínez-Ripoll, J. K. Zhu, A. Albert, The structure of Arabidopsis thaliana OST1 provides insights into the kinase regulation mechanism in response to osmotic stress. J. Mol. Biol. 414, 135-144 (2011).

39. J. Zhang et al., Identification of SLAC1 anion channel residues required for $\mathrm{CO}_{2} / \mathrm{bi}-$ carbonate sensing and regulation of stomatal movements. Proc. Natl. Acad. Sci. U.S.A 115, 11129-11137 (2018).

40. Y. Takahashi et al., MAP3Kinase-dependent SnRK2-kinase activation is required for abscisic acid signal transduction and rapid osmotic stress response. Nat. Commun. 11, 12 (2020).

41. S. Törnroth-Horsefield et al., Structural mechanism of plant aquaporin gating. Nature 439, 688-694 (2006)

42. Z. Zhang, F. Liu, J. Chen, Conformational changes of CFTR upon phosphorylation and ATP binding. Cell 170, 483-491.e8 (2017).

43. J. Sun et al., Crystal structure of the plant dual-affinity nitrate transporter NRT1.1. Nature 507, 73-77 (2014).

44. J. L. Parker, S. Newstead, Molecular basis of nitrate uptake by the plant nitrate transporter NRT1.1. Nature 507, 68-72 (2014).

45. Y. Yamamoto et al., The transmembrane region of guard cell SLAC1 channels perceives $\mathrm{CO}_{2}$ signals via an ABA-independent pathway in Arabidopsis. Plant Cell 28 557-567 (2016). 